Pacific Journal of Mathematics

TAUBERIAN THEOREMS BETWEEN THE LOGARITHMIC 


\section{TAUBERIAN THEOREMS BETWEEN THE LOGARITHMIC AND ABEL-TYPE SUMMABILITY METHODS}

\section{David Borwein aNd BRUCE WATSON}

The object of this paper is to show that if a series is summable by the logarithmic method $L$, then the series is also summable by the Abel method $A_{\lambda}$, provided a tauberian condition of the "slowly decreasing" type is satisfied.

1. Introduction. Suppose throughout that $\left\{s_{n}\right\}$ is a sequence of numbers, $\lambda$ real is real, $\varepsilon_{0}^{\lambda}=1, \varepsilon_{n}^{\lambda}=\left(\begin{array}{c}n+\lambda \\ n\end{array}\right)$ for $n=1,2,3, \cdots$, and

$$
v_{n}^{\lambda}=\frac{\varepsilon_{n}^{\lambda} \Gamma(\lambda+1)}{(n+1)^{\lambda}} \text { for } n=0,1,2, \cdots \text {. }
$$

We are concerned with the methods of summability $A_{\lambda}$ introduced and studied by Borwein [1] and the logarithmic method $L$. They are defined as follows. Let

$$
\begin{aligned}
\sigma_{\lambda}(y) & =(1+y)^{-\lambda-1} \sum_{n=0}^{\infty} \varepsilon_{n}^{\lambda} s_{n}\left(\frac{y}{1+y}\right)^{n}, \text { and } \\
L(y) & =\frac{1}{\log (1+y)} \sum_{n=0}^{\infty} \frac{s_{n}}{n+1}\left(\frac{y}{1+y}\right)^{n+1} .
\end{aligned}
$$

If $\sigma_{\lambda}(y)$ converges for $y>0$ and tends to $s$ as $y \rightarrow \infty$, then we say that the sequence $\left\{s_{n}\right\}$ is $A_{\lambda}$-convergent to $s$ and write $s_{n} \rightarrow s\left(A_{\lambda}\right)$. The method $A_{0}$ is the ordinary Abel method.

If $L(y)$ converges for $y>0$ and tends to $s$ as $y \rightarrow \infty$, then we say that $\left\{s_{n}\right\}$ is $L$-convergent to $s$ and write $s_{n} \rightarrow s(L)$.

Evidently, $s_{n} \rightarrow s(L)$ if and only if

$$
-\frac{1}{\log (1-x)} \sum_{n=0}^{\infty} \frac{s_{n}}{n+1} x^{n+1}
$$

converges for $0<x<1$ and tends to $s$ as $x \rightarrow 1^{-}$.

LEMMA 1. $A_{\lambda}$ is regular for $\lambda>-1$. [That is, $s_{n} \rightarrow s$ implies $\left.s_{n} \rightarrow s\left(A_{2}\right)\right]$.

LEMma 2. $L$ is regular.

Lemma 3. $A_{\lambda+\varepsilon} \subset A_{\lambda}$ for $\lambda>-1$, and $\varepsilon>0$. [That is, $s_{n} \rightarrow$ $s\left(A_{\lambda+\varepsilon}\right)$ implies $s_{n} \rightarrow s\left(A_{2}\right)$ and there exists a sequence $\left\{s_{n}\right\}$, depending on $\lambda$ and $\varepsilon$, such that $\left\{s_{n}\right\}$ is $A_{\lambda}$-convergent but not $A_{\lambda+\varepsilon}$-convergent.] 
LemMA 4. $A_{\lambda} \subset L$ for $\lambda>-1$.

Lemmas 1 and 3 were established by Borwein in [1]. Lemma 4 was proved by Borwein in [2] as a particular case of a more general inclusion theorem on methods of summability based on power series. Lemma 2 is a standard result found, for example, in [4].

2. The main theorem. Suppose that $\Phi$ is a nonnegative, continuous, strictly increasing function on $[a, \infty)$, for some $a$, such that $\Phi(t) \rightarrow \infty$ as $t \rightarrow \infty$.

The real-valued function $f$ is said to be slowly decreasing with respect to $\Phi$ if $\lim \inf \{f(y)-f(x)\} \geqq 0$ whenever $y \geqq x \rightarrow \infty$ and $\Phi(y)-\Phi(x) \rightarrow 0$.

THEOREM 1. For $\lambda>-1$, if $s_{n} \rightarrow s(L)$ and $\sigma_{\lambda}(t)$ is slowly decreasing with respect to $\log \log t$, then $s_{n} \rightarrow s\left(A_{\lambda}\right)$.

In connection with the methods $A_{\lambda}$, we proved the following lemma in [3].

LEMMA 5. For $\lambda>-1$ and $\varepsilon>0$, if $s_{n} \rightarrow s\left(A_{\lambda}\right)$ and $\sigma_{\lambda+\varepsilon}(t)$ is slowly decreasing with respect to $\log t$, then $s_{n} \rightarrow s\left(A_{\lambda+\varepsilon}\right)$.

3. Methods of summability based on power series. Suppose that $p_{n} \geqq 0, q_{n} \geqq 0, \sum_{v=n}^{\infty} p_{v}>0$, and $\sum_{v=n}^{\infty} q_{v}>0$ for $n=0,1,2, \cdots$. Set

$$
\begin{aligned}
& p(x)=\sum_{n=0}^{\infty} p_{n} x^{n}, \quad \text { and } \\
& q(x)=\sum_{n=0}^{\infty} q_{n} x^{n} .
\end{aligned}
$$

Let $\rho_{p}$ and $\rho_{q}$ denote their respective radii of convergence. We also write

$$
\begin{aligned}
& p_{s}(x)=\frac{1}{p(x)} \sum_{n=0}^{\infty} p_{n} s_{n} x^{n} \\
& q_{s}(x)=\frac{1}{q(x)} \sum_{n=0}^{\infty} q_{n} s_{n} x^{n} .
\end{aligned}
$$

The power series method $P$ is defined as follows. If $\rho_{p}>0$, $\sum_{n=0}^{\infty} p_{n} s_{n} x^{n}$ converges for $0<x<\rho_{p}$ and $\lim _{x \rightarrow \rho_{p}}-p_{s}(x)=s$, then we write $s_{n} \rightarrow s(P)$.

The method $Q$ is defined similarly. 
Borwein has proved [2] the following lemma.

LEMma 6. (i) If $0<\rho_{p}<\infty$, then a necessary and sufficient condition for $P$ to be regular is that $\sum_{n=0}^{\infty} p_{n}\left(\rho_{p}\right)^{n}=\infty$.

(ii) If $\rho_{p}=\infty$ then $P$ is regular.

Suppose that $\chi(t)$ is a function of bounded variation on $[0,1]$, and $\chi^{*}(t)$ is its associated normalized function. That is,

$$
\chi^{*}(t)= \begin{cases}0 & t=0 \\ \frac{1}{2}\{\chi(t+)+\chi(t-)\}-\chi(0) & 0<t<1 \\ \chi(1)-\chi(0) & t=1 .\end{cases}
$$

A sequence $\left\{\mu_{n}\right\}$ is called an $m$-sequence if, for some $\chi$,

$$
\mu_{n}=\int_{0}^{1} t^{n} d \chi(t) \text { for } n=0,1,2, \cdots \text {. }
$$

If, in addition,

$$
\mu_{n} \geqq \delta \int_{0}^{1} t^{n}\left|d \chi^{*}(t)\right| \text { for } 0<\delta \leqq 1 \text { and }
$$

$n=N, N+1, \cdots$, then $\left\{\mu_{n}\right\}$ is called an $\bar{m}$-sequence.

LEMMA 7. If $p_{n}=\mu_{n} q_{n}(n=N, N+1, \cdots),\left\{\mu_{n}\right\}$ is an $\bar{m}$-sequence, $\rho_{p}=\rho_{q}>0$, and $P$ is regular, then $Q \subseteq P$. (That is, $s_{n} \rightarrow s(Q)$ implies $s_{n} \rightarrow s(P)$.)

This result is due to Borwein (see [2], Theorem A').

We require the following two lemmas.

LEMMA 8. An m-sequence which converges to a positive limit is an $\bar{m}$-sequence.

LEMMA 9. The sequences $\left\{v_{n}^{2}\right\}$ and $\left\{1 / v_{n}^{2}\right\}$ are $\bar{m}$-sequences for $\lambda>-1$.

The proof of Lemma 8 is straightforward and Lemma 9 was established in [4], Theorem 211.

The next result is used in the proof of Theorem 1.

THEOREM 2. Let $Q$ be a regular power series method and suppose that $\left\{\mu_{n}\right\}$ is an $\bar{m}$-sequence such that $\mu_{n} \rightarrow a>0$. Then $\mu_{n} s_{n} \rightarrow a s(Q)$ 
whenever $s_{n} \rightarrow s(Q)$.

Proof. Suppose that $s_{n} \rightarrow s(Q)$. Set $p_{n}=\mu_{n} q_{n}$ for $n=0,1,2, \cdots$. Since $\mu_{n} \geqq 0$ and $\mu_{n} \rightarrow a$ it is easy to verify that $\rho_{p}=\rho_{q}$. If $\rho_{p}=$ $\infty$, then $P$ is regular by Lemma 6(ii). Otherwise, since $p_{n} \sim a q_{n}, P$ is regular by Lemma $6(\mathrm{i})$.

Therefore, by Lemma $7, s_{n} \rightarrow s(P)$. That is,

$$
\frac{1}{p(x)} \sum_{n=0}^{\infty} s_{n} \mu_{n} q_{n} x^{n} \longrightarrow s \text { as } x \longrightarrow \rho_{P}^{-} \text {. }
$$

In addition, since $Q$ is regular,

$$
\frac{p(x)}{q(x)}=\frac{1}{q(x)} \sum_{n=0}^{\infty} \mu_{n} q_{n} x^{n} \longrightarrow a \quad \text { as } \quad x \longrightarrow \rho_{q}^{-} .
$$

Application of $Q$ to $\left\{\mu_{n} s_{n}\right\}$ yields

$$
\begin{aligned}
\frac{1}{q(x)} \sum_{n=0}^{\infty} \mu_{n} s_{n} q_{n} x^{n} & \\
= & \frac{p(x)}{q(x)} \frac{1}{p(x)} \sum_{n=0}^{\infty} s_{n} \mu_{n} q_{n} x^{n} \\
& \longrightarrow \text { as as } x \longrightarrow \rho_{q}^{-}=\rho_{p}^{-} \text {by (3) and (4). }
\end{aligned}
$$

This completes the proof.

CoRollary to THEOREM 2. $s_{n} \rightarrow s(L)$ if and only if $v_{n}^{\lambda} s_{n} \rightarrow s(L)$.

This is immediate in view of Lemmas 8 and 9, and the fact that $v_{n}^{\lambda} \rightarrow 1$ as $n \rightarrow \infty$.

4. An integral transformation. The integral transformation $J_{\lambda}(w)$ of the function $f(t)$, for $\lambda>-1$ and $w>0$, is defined as follows.

$$
J_{\lambda}(w)=\frac{1}{\log (1+w)} \int_{0}^{w}(1+t)^{\lambda-1}\left(\log \frac{w(1+t)}{t(1+w)}\right)^{\lambda} f(t) d t .
$$

THEOREM 3. If $\lambda>-1$ and $f(t)=\sigma_{\lambda}(t)$ is convergent for all $t>0$, then $J_{\lambda}(w) \rightarrow s$ as $w \rightarrow \infty$ if and only if $s_{n} \rightarrow s(L)$.

Proof. Setting $u=(t(1+w)) /(w(1+t))$ in $J_{\lambda}(w)$ gives

$$
\begin{aligned}
& J_{\lambda}(w) \\
& =\frac{1}{\log (1+w)} \int_{0}^{w}(1+t)^{\lambda-1}\left(\log \frac{w(1+t)}{t(1+w)}\right)^{\lambda}(1+t)^{-\lambda-1} \sum_{n=0}^{\infty} \varepsilon_{n}^{\lambda} s_{n}\left(\frac{t}{1+t}\right)^{n} d t
\end{aligned}
$$




$$
\begin{aligned}
& =\frac{1}{\log (1+w)} \int_{0}^{1} \sum_{n=0}^{\infty} \varepsilon_{n}^{\lambda} s_{n}\left(\frac{w}{1+w}\right)^{n+1} u^{n}\left(\log \frac{1}{u}\right)^{\lambda} d u \\
& =\frac{1}{\log (1+w)} \sum_{n=0}^{\infty} \varepsilon_{n}^{\lambda} s_{n}\left(\frac{w}{1+w}\right)^{n+1} \int_{0}^{1} u^{n}\left(\log \frac{1}{u}\right)^{\lambda} d u \\
& =\frac{\Gamma(\lambda+1)}{\log (1+w)} \sum_{n=0}^{\infty} \frac{\varepsilon_{n}^{\lambda}}{(n+1)^{\lambda+1}} s_{n}\left(\frac{w}{1+w}\right)^{n+1} \\
& =\frac{1}{\log (1+w)} \sum_{n=0}^{\infty} \frac{v_{n}^{\lambda} s_{n}}{n+1}\left(\frac{w}{1+w}\right)^{n+1} .
\end{aligned}
$$

The convergence, for $t>0$, of the series defining $\sigma_{2}(t)$ implies its absolute convergence. This justifies the integration term by term and, in view of the corollary to Theorem 2, the proof is complete.

\section{Additional lemmas.}

LEMMA 10. For $\lambda>-1, \sum_{n=0}^{\infty} \varepsilon_{n}^{\lambda} s_{n} x^{n}$ is absolutely convergent for $|x|<1$ if and only if $\sum_{n=0}^{\infty}\left(s_{n} /(n+1)\right) x^{n}$ is absolutely convergent for $|x|<1$.

We omit the simple proof.

Lemma 11. For $0<t<w$,

$$
\log \frac{w(1+t)}{t(1+w)}>\frac{w-t}{w(1+t)} .
$$

Proof. For $x>1$,

$$
\log x=\log x-\log 1=\frac{x-1}{\theta}>\frac{x-1}{x}
$$

where $1<\theta<x$. The result follows by observing that, for $0<t<$ $w, x=(w(1+t)) /(t(1+w))>1$.

LemMa 12. For fixed $\gamma>1$ and $\lambda>-1$,

$$
\begin{aligned}
I(x) & =\int_{0}^{x}(1+t)^{\lambda-1}\left(\left(\log \frac{x^{\gamma}(1+t)}{t\left(1+x^{r}\right)}\right)^{\lambda}-\left(\log \frac{x(1+t)}{t(1+x)}\right)^{\lambda}\right) d t \\
& =O(1) .
\end{aligned}
$$

Proof. Suppose $\lambda \geqq 1$. Then, for $x \geqq 1$,

$$
\begin{aligned}
|I(x)| & =I(x) \\
& \leqq \lambda \log \frac{x^{\gamma}(1+x)}{x\left(1+x^{\gamma}\right)} \int_{0}^{x}(1+t)^{\lambda-1}\left(\log \frac{x^{\gamma}(1+t)}{t\left(1+x^{\gamma}\right)}\right)^{\lambda-1} d t
\end{aligned}
$$




$$
\begin{aligned}
& \leqq \lambda \log \frac{x^{\gamma}(1+x)}{x\left(1+x^{\gamma}\right)}\left(\int_{0}^{1}+\int_{1}^{x}\right)(1+t)^{\lambda-1}\left(\log \frac{1+t}{t}\right)^{\lambda-1} d t \\
& =I_{1}(x)+I_{2}(x) .
\end{aligned}
$$

Now,

$$
\int_{0}^{1}(1+t)^{\lambda-1}\left(\log \frac{1+t}{t}\right)^{\lambda-1} d t<\infty
$$

Hence,

$$
I_{1}(x)=O(1)
$$

Also,

$$
\begin{aligned}
I_{2}(x) & =O(1) \log \frac{x^{\gamma}(1+x)}{x\left(1+x^{\gamma}\right)} \int_{1}^{x} d t \\
& =O(1) x \log \frac{1+x}{x}=O(1) .
\end{aligned}
$$

Suppose $0<\lambda<1$. By Lemma 11 we have,

$$
\begin{aligned}
|I(x)| & =I(x) \\
& \leqq \lambda \log \frac{x^{r}(1+x)}{x\left(1+x^{r}\right)} \int_{0}^{x}(1+t)^{\lambda-1}\left(\log \frac{x(1+t)}{t(1+x)}\right)^{\lambda-1} d t \\
& <\lambda \frac{M}{x} \int_{0}^{x}(1+t)^{\lambda-1}\left(\frac{x-t}{x(1+t)}\right)^{\lambda-1} d t
\end{aligned}
$$

since $x \log \left(x^{\gamma}(1+x)\right) /\left(x\left(1+x^{r}\right)\right) \leqq M$.

Therefore

$$
I(x) \leqq \lambda \frac{M}{x^{\lambda}} \int_{0}^{\infty}(x-t)^{\lambda-1} d t=M .
$$

Suppose $-1<\lambda<0$. Then

$$
\begin{aligned}
|I(x)| & =-I(x) \\
& =\left(\int_{0}^{x / 2}+\int_{x / 2}^{x}\right)(1+t)^{\lambda-1}\left(\left(\log \frac{x(1+t)}{t(1+x)}\right)^{\lambda}-\left(\log \frac{x^{\gamma}(1+t)}{t\left(1+x^{\lambda}\right)}\right)^{\lambda}\right) d t \\
& =I_{1}(x)+I_{2}(x) .
\end{aligned}
$$

Using Lemma 11 and the fact that

$$
\left|x \log \frac{x\left(1+x^{r}\right)}{(1+x) x^{r}}\right| \leqq M
$$

we have 


$$
\begin{aligned}
0 \leqq I_{1}(x) & \leqq \lambda\left(\log \frac{x\left(1+x^{\gamma}\right)}{x^{\gamma}(1+x)}\right) \int_{0}^{x / 2}(1+t)^{\lambda-1}\left(\log \frac{x(1+t)}{t(1+x)}\right)^{\lambda-1} d t \\
& \leqq-\frac{\lambda M}{x} \int_{0}^{x / 2}(1+t)^{\lambda-1}\left(\frac{x-t}{x(1+t)}\right)^{\lambda-1} d t \\
& =M\left((1 / 2)^{\lambda}-1\right) .
\end{aligned}
$$

For $I_{2}(x)$, since $1+t>x / 2$,

$$
\begin{aligned}
0 & \leqq I_{2}(x) \leqq \int_{x / 2}^{x}(1+t)^{\lambda-1}\left(\log \frac{x(1+t)}{t(1+x)}\right)^{\lambda} d t \\
& \leqq \int_{x / 2}^{x}(1+t)^{\lambda-1}\left(\frac{x-t}{x(1+t)}\right)^{\lambda} d t \\
& =\frac{1}{x^{\lambda}} \int_{x / 2}^{x}(x-t)^{\lambda} \frac{d t}{1+t} \\
& \leqq \frac{2}{x^{\lambda+1}} \int_{x / 2}^{x}(x-t)^{\lambda} d t \\
& =\frac{1}{(\lambda+1) 2^{\lambda}} .
\end{aligned}
$$

Hence, $I(x)=O(1)$ in this case.

Finally, since the case $\lambda=0$ is trivial, the lemma is established.

Lemma 13. For $\gamma>1$, and $\lambda>-1$,

$$
\begin{aligned}
\int_{x}^{x^{\lambda}}(1 & +t)^{\lambda-1}\left(\log \frac{x^{\gamma}(1+t)}{t\left(1+x^{\gamma}\right)}\right)^{\lambda} d t \\
& =(\gamma-1) \log (1+x)+o(\log (1+x)) .
\end{aligned}
$$

Proof. Set $\left\{s_{n}\right\}=\{1\}$. Then $\sigma_{\lambda}(t)=1$ and, by Theorem 3, putting $f(t)=\sigma_{\lambda}(t)$ in (5) gives

$$
J_{\lambda}(x)=1+o(1) \text { as } x \longrightarrow \infty .
$$

Now by Lemma 12 ,

$$
\begin{aligned}
\int_{x}^{x^{\lambda}}(1+t)^{\lambda-1}\left(\log \frac{x^{\gamma}(1+t)}{t\left(1+x^{\gamma}\right)}\right)^{\lambda} d t \\
=\left(\int_{0}^{x^{\lambda}}-\int_{0}^{x}\right)(1+t)^{\lambda-1}\left(\log \frac{x^{\gamma}(1+t)}{t\left(1+x^{\gamma}\right)}\right)^{\lambda} d t \\
=\log \left(1+x^{\gamma}\right)+o\left(\log \left(1+x^{\gamma}\right)\right)-\log (1+x)+o(\log (1+x)) \\
\quad+o(1) \\
=(\gamma-1) \log (1+x)+o(\log (1+x)) .
\end{aligned}
$$

This establishes the lemma. 
6. A general tauberian result.

THEOREM 4. Suppose that the following conditions hold:

(6) $K(w, t)$ is defined, real-valued, and nonnegative for $w>0, t \geqq$ 0 ; moreover, $\int_{0}^{\infty} K(w, t) d t$ exists in the sense of Lebesgue for each $w>0$,

$$
\int_{0}^{\infty} K(w, t) d t \longrightarrow 1 \text { as } w \longrightarrow \infty,
$$

(8) $f$ is real-valued and continuous on $(0, \infty)$,

(9) $F(w)=\int_{0}^{\infty} K(w, t) f(t) d t$ exists in the Cauchy-Lebesgue sense for each $w>0$,

(10) $\liminf \{f(y)-f(x)\} \geqq-\mu$ for some fixed finite nonnegative $\mu$, whenever $y \geqq x \rightarrow \infty$ and $\Phi(y)-\Phi(x) \rightarrow 0$,

$$
\Phi(x)-\Phi(x-1) \longrightarrow 0 \text { as } x \longrightarrow \infty,
$$

$$
\begin{aligned}
& \int_{0}^{\infty} K(w, t) d t \longrightarrow 0 \quad \text { whenever } w>x \longrightarrow \infty \text { and } \\
& \Phi(w)-\Phi(x) \longrightarrow \infty
\end{aligned}
$$

$$
\begin{aligned}
& \int_{x}^{\infty} K(w, t)(\Phi(t)-\Phi(x)) d t \longrightarrow 0 \text { whenever } \\
& x>w \longrightarrow \infty \text { and } \Phi(x)-\Phi(w) \longrightarrow \infty, \text { and }
\end{aligned}
$$$$
F(w)=O(1) \text { for } w>0 .
$$

Then $f(t)=O(1)$ for $t>0$.

This result was established in [5]. A version of this theorem with (10) replaced by the stronger condition that $f$ be slowly decreasing with respect to $\Phi$ can be found in [3]. The proofs are very similar.

7. A theorem on boundedness. In this section we deduce a weakened form of Theorem 1 from the general tauberian result of $\S 6$.

THEOREM 5. If $\lambda>-1, \infty>\mu \geqq 0, s_{n} \rightarrow s(L)$, and $\liminf \left\{\sigma_{\lambda}(y)-\right.$ $\left.\sigma_{\lambda}(x)\right\} \geqq-\mu$ whenever $y \geqq x \rightarrow \infty$ and $\Phi(y)-\Phi(x) \rightarrow 0$, then $\sigma_{\lambda}(t)=$ $O(1)$. 


$$
\begin{aligned}
K(w, t) & = \begin{cases}\frac{1}{\log (1+w)}(1+t)^{\lambda-1}\left(\log \frac{w(1+t)}{t(1+w)}\right)^{\lambda} 0<t<w \\
0 & \text { otherwise },\end{cases} \\
\Phi(t) & = \begin{cases}t / e^{e} & 0 \leqq t<e^{e} \\
\log \log t & e^{e} \leqq t,\end{cases}
\end{aligned}
$$

and

$$
f(t)=\sigma_{\lambda}(t)
$$

First, note that if $\left\{s_{n}\right\}=\{1\}$, then $s_{n} \rightarrow 1(L)$ and $\sigma_{\lambda}(t)=1$. Hence, by Theorem 3 with $f(t)=\sigma_{\lambda}(t)=1$ in (5), we have

$$
\begin{aligned}
& \int_{0}^{\infty} K(w, t) d t \\
& \quad=\frac{1}{\log (1+w)} \int_{0}^{w}(1+t)^{\lambda-1}\left(\log \frac{w(1+t)}{t(1+w)}\right)^{\lambda} d t \\
& \quad=J_{\lambda}(w) \longrightarrow 1 \text { as } w \longrightarrow \infty .
\end{aligned}
$$

This establishes (6) and (7).

Conditions (8), (9), (10) and (14) hold by hypotheses, and (11) clearly holds.

Furthermore, condition (13) is immediate since $K(w, t)=0$ whenever $t \geqq w$. It remains to show (12). Suppose $-1<\lambda<0$. Then, by Lemma 11, we have

$$
\begin{aligned}
\int_{0}^{x} K(w, t) d t \\
\quad=\frac{1}{\log (1+w)} \int_{0}^{x}(1+t)^{\lambda-1}\left(\log \frac{w(1+t)}{t(1+w)}\right)^{\lambda} d t \\
\quad \leqq \frac{1}{\log (1+w)} \int_{0}^{x}(1+t)^{\lambda-1}\left(\frac{w-t}{w(1+t)}\right)^{\lambda} d t \\
\quad=\frac{1}{\log (1+w)} \int_{0}^{x}(1-t / w)^{\lambda} \frac{d t}{1+t} \\
\quad \leqq \frac{(1-x / w)^{\lambda}}{\log (1+w)} \int_{0}^{x} \frac{d t}{1+t} \\
\quad=(1-x / w)^{\lambda} \frac{\log (1+x)}{\log (1+w)}=o(1)
\end{aligned}
$$

as $w>x \rightarrow \infty$ and $\log \log w-\log \log x \rightarrow \infty$, since the latter implies $\log x / \log w \rightarrow 0$ and $x / w \rightarrow 0$.

Suppose $\lambda \geqq 0$ and $x>1$. Then 


$$
\begin{aligned}
\log (1+w) \int_{0}^{x} K(w, t) d t & =\int_{0}^{x}(1+t)^{\lambda-1}\left(\log \frac{w(1+t)}{t(1+w)}\right)^{\lambda} d t \\
& \leqq\left(\int_{0}^{1}+\int_{1}^{x}\right)(1+t)^{\lambda-1}\left(\log \frac{1+t}{t}\right)^{\lambda} d t \\
& =I_{1}+I_{2} .
\end{aligned}
$$

Setting $u=1 / t$ in $I_{1}$ gives

$$
\begin{aligned}
I_{1} & =\int_{1}^{\infty}(1+1 / u)^{\lambda-1}(\log (1+u))^{\lambda} \frac{d u}{u^{2}} \\
& =O(1) .
\end{aligned}
$$

Furthermore,

$$
\begin{aligned}
I_{2} & =O(1) \int_{1}^{x}(1+t)^{-1} d t \\
& =O(1) \log (1+x)-O(1) .
\end{aligned}
$$

Therefore,

$$
\begin{aligned}
& \int_{0}^{x} K(w, t) d t \\
& \quad=\frac{1}{\log (1+w)}\left\{I_{1}+I_{2}\right\} \\
& \quad=o(1)+O(1) \frac{\log (1+x)}{\log (1+w)}=o(1)
\end{aligned}
$$

as $w>x \rightarrow \infty$ and $\log \log w-\log \log x \rightarrow \infty$.

This completes the proof.

8. Proof of Theorem 1. Assign $\varepsilon>0$. Since $\sigma_{\lambda}(t)$ is slowly decreasing with respect to $\Phi(t)=\log \log t$, there exist positive numbers $X$ and $\delta$ such that $\sigma_{\lambda}(y)-\sigma_{\lambda}(x)>-\varepsilon$ whenever $y>x>X$ and $\log \log y-\log \log x<\delta$; or equivalently, writing $\delta=\log \gamma$

$$
\sigma_{\lambda}(x)-\varepsilon<\sigma_{\lambda}(y) \text { whenever } X<x<y<x^{\gamma} .
$$

Suppose, without loss of generality, that $s=0$. Then $J_{\lambda}(w) \rightarrow 0$ as $w \rightarrow \infty$.

Relation (15) implies, for $x>X$, that

$$
\begin{aligned}
I_{1} & =\int_{x}^{x^{\lambda}}(1+t)^{\lambda-1}\left(\log \frac{x^{\gamma}(1+t)}{t\left(1+x^{\gamma}\right)}\right)^{\lambda}\left(\sigma_{\lambda}(x)-\varepsilon\right) d t \\
& \leqq \int_{x}^{x \widetilde{\gamma}}(1+t)^{\lambda-1}\left(\log \frac{x^{\gamma}(1+t)}{t\left(1+x^{r}\right)}\right)^{\lambda} \sigma_{\lambda}(t) d t \\
& =I_{2} .
\end{aligned}
$$


Now, by Theorem 5 and Lemma 12 ,

$$
\begin{aligned}
I_{2} & =\left(\int_{0}^{x \gamma}-\int_{0}^{x}\right)(1+t)^{\lambda-1}\left(\log \frac{x^{\gamma}(1+t)}{t\left(1+x^{\gamma}\right)}\right)^{\lambda} \sigma_{\lambda}(t) d t \\
& =\log \left(1+x^{\gamma}\right) J_{\lambda}\left(x^{\gamma}\right)-\log (1+x) J_{\lambda}(x)+O(1) \\
& =o\left(\log \left(1+x^{\gamma}\right)\right)+o(\log (1+x)) \\
& =o(\log (1+x)) .
\end{aligned}
$$

By Lemma 13,

$$
\begin{aligned}
I_{1} & =\left(\sigma_{\lambda}(x)-\varepsilon\right) \int_{x}^{x^{\gamma}}(1+t)^{\lambda-1}\left(\log \frac{x^{\gamma}(1+t)}{t\left(1+x^{\gamma}\right)}\right)^{\lambda} d t \\
& =\left(\sigma_{\lambda}(x)-\varepsilon\right)((\gamma-1) \log (1+x)+o(\log (1+x))) .
\end{aligned}
$$

But $I_{1} \leqq I_{2}$ implies

$$
\sigma_{\lambda}(x)-\varepsilon \leqq \frac{o(1)}{(\gamma-1)+o(1)} .
$$

Therefore,

$$
\limsup _{x \rightarrow \infty} \sigma_{\lambda}(x) \leqq \varepsilon .
$$

In a similar fashion, we can show that

$$
-\varepsilon \leqq \liminf _{x \rightarrow \infty} \sigma_{\lambda}(x) .
$$

Combining (16) and (17) completes the proof of theorem.

9. A counterexample. In this section we give an example which shows that Theorem 1 would be false if $\log \log t$ were replaced by $\log t$. That is, a more delicate tauberian condition on $\sigma_{\lambda}(t)$ is required than what is obtained by using the standard definition of slowly decreasing.

Lemma 14. If $f(x)$ is absolutely continuous on $[0, T]$ for each $T>0$ and $f^{\prime}(x)>-M / x$ for all $x>0$, then $f(x)$ is slowly decreasing with respect to $\log x$.

Proof. Assign $\varepsilon>0$. Then if $y>x>0$

$$
\begin{aligned}
f(y)-f(x) & =\int_{x}^{y} f^{\prime}(t) d t \\
& >-M \int_{x}^{y} \frac{1}{t} d t \\
& =-M(\log y-\log x)>-\varepsilon
\end{aligned}
$$


whenever $\log y-\log x<\varepsilon / M$. This completes the proof.

THEOREM 6. There exists a sequence $\left\{s_{n}\right\}$ such that $s_{n} \rightarrow s(L)$ and, for every $\lambda>-1, \sigma_{\lambda}(t)$ is slowly decreasing with respect to $\log t$, but $\left\{s_{n}\right\}$ is not $A_{\lambda}$-convergent.

Proof. Let $\left\{s_{n}\right\}$ be the real part of the sequence $\left\{\varepsilon_{n}^{i}\right\}$. For any $\lambda>-1, \sigma_{\lambda}(t)$ exists for $t>0$, and we have

$$
\varepsilon_{n}^{i}=\frac{\Gamma(\lambda+i+1)}{\Gamma(\lambda+1) \Gamma(i+1)} \frac{\varepsilon_{n}^{\lambda+1}}{\varepsilon_{n}^{\lambda}}+o(1) .
$$

Therefore, $\sigma_{\lambda}(t)$ is the real part of

$$
\begin{aligned}
& (1+t)^{-\lambda-1} \sum_{n=0}^{\infty} \frac{\Gamma(\lambda+i+1)}{\Gamma(\lambda+1) \Gamma(i+1)} \varepsilon_{n}^{\lambda+i}\left(\frac{t}{1+t}\right)^{n}+(1+t)^{-\lambda-1} \sum_{n=0}^{\infty} \varepsilon_{n}^{\lambda} o(1)\left(\frac{t}{1+t}\right)^{n} \\
& =\frac{\Gamma(\lambda+i+1)}{\Gamma(\lambda+1) \Gamma(i+1)}(1+t)^{i}+o(1) .
\end{aligned}
$$

The first term above has a derivative which is $O(1 / t)$ and, hence, the real part of the first term has a derivative which is $O(1 / t)$. The second term is $o(1)$ since $A_{\lambda}$ is regular. Hence, the real part of this term is slowly decreasing with respect to any $\Phi$. Therefore, by Lemma $14, \sigma_{\lambda}(t)$ is slowly decreasing with respect to $\log t$.

Next, it is clear that $\left\{s_{n}\right\}$ is not $A_{2}$-convergent.

However,

$$
\begin{aligned}
J_{0}(w) & =\frac{1}{\log (1+w)} \int_{0}^{w}(1+t)^{-1} \sigma_{0}(t) d t \\
& =\frac{1}{\log (1+w)} \int_{0}^{w} \frac{\cos \log (1+t)}{1+t} d t \\
& =\frac{\sin \log (1+w)}{\log (1+w)} \longrightarrow 0 \text { as } w \longrightarrow \infty .
\end{aligned}
$$

Hence, by Theorem 3, $s_{n} \rightarrow O(L)$. This completes the proof.

\section{REFERENCES}

1. D. Borwein, On a scale of Abel-type summability methods, Proc. Cambridge Phil. Soc., 53 (1957), 318-322.

2. - On methods of summability based on power series, Proc. Royal Soc. Edinburgh, 64 (1957), 342-349.

3. D. Borwein and B. Watson, Tauberian theorems on a scale of Abel-type summability methods, Journal Fur Die Reine Und Angewandte Mathematik, 298 (1978), 1-7.

4. G. H. Hardy, Divergent Series, Oxford, 1949.

5. B. Watson, Tauberian theorems on a scale of Abel-type summability methods, $\mathrm{Ph} . \mathrm{D}$. Thesis, The University of Western Ontario, 1974. 
Received July 30, 1979 and in revised form June 2, 1981. Supported in part by the Natural Sciences and Engineering Research Council of Canada, Grants A.2983 and A. 4646.

The University of Western Ontario

London, Ontario, Canada N6A 5B7

AND

Memorial University of Newfoundland

St. John's, Newfoundland, Canada A1B $3 \mathrm{X} 7$ 



\section{PACIFIC JOURNAL OF MATHEMATICS}

\section{EDITORS}

DONALD BABBITT (Managing Editor)

J. DUGUNDJI

University of California

Los Angeles, California 90024

Hugo Rossi

University of Utah

Salt Lake City, UT 84112

C. C. Moore and Arthur Agus

Department of Mathematics

University of Southern California

Los Angeles, California 90007

R. FinN and J. Milgram

Stanford University

Stanford, California 94305

University of California

Berkeley, CA 94720

\section{ASSOCIATE EDITORS}
R. ARNES
E. F. BeCKenBaCH
B. H. NEUMANN
F. WoLF
K. YoshidA

\section{SUPPORTING INSTITUTIONS}

UNIVERSITY OF ARIZONA

UNIVERSITY OF BRITISH COLUMBIA

CALIFORNIA INSTITUTE OF TECHNOLOGY

UNIVERSITY OF CALIFORNIA

MONTANA STATE UNIVERSITY

UNIVERSITY OF NEVADA, RENO

NEW MEXICO STATE UNIVERSITY

OREGON STATE UNIVERSITY
UNIVERSITY OF OREGON

UNIVERSITY OF SOUTHERN CALIFORNIA

STANFORD UNIVERSITY

UNIVERSITY OF HAWAII

UNIVERSITY OF TOKYO

UNIVERSITY OF UTAH

WASHINGTON STATE UNIVERSITY

UNIVERSITY OF WASHINGTON 


\section{Pacific Journal of Mathematics}

\section{Vol. 101, No. $1 \quad$ November, 1982}

Natália Bebiano, On the evaluation of permanents $\ldots \ldots \ldots \ldots \ldots \ldots \ldots \ldots$

David Borwein and Bruce Brigham Watson, Tauberian theorems between

the logarithmic and Abel-type summability methods $\ldots \ldots \ldots \ldots \ldots \ldots 11$

Leo George Chouinard, II, Hermite semigroup rings $\ldots \ldots \ldots \ldots \ldots \ldots \ldots$

Kun-Jen Chung, Remarks on nonlinear contractions $\ldots \ldots \ldots \ldots \ldots \ldots . \ldots 4$

Lawrence Jay Corwin, Representations of division algebras over local

fields. II ......................................... 49

Mahlon M. Day, Left thick to left lumpy—a guided tour $\ldots \ldots \ldots \ldots \ldots 71$

M. Edelstein and Mo Tak Kiang, On ultimately nonexpansive

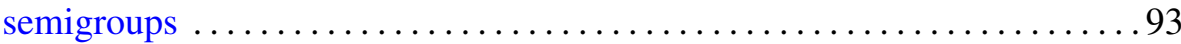

Mary Rodriguez Embry, Semigroups of quasinormal operators . ........ 103

William Goldman and Morris William Hirsch, Polynomial forms on

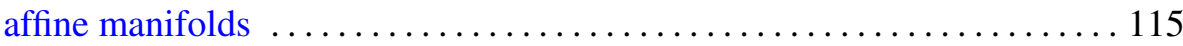

S. Janakiraman and T. Soundararajan, Totally bounded group topologies

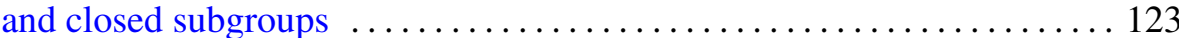

John Rowlay Martin, Lex Gerard Oversteegen and Edward D.

Tymchatyn, Fixed point set of products and cones $\ldots \ldots \ldots \ldots \ldots \ldots 133$

Jan van Mill, A homogeneous Eberlein compact space which is not metrizable ........................................ 141

Steven Paul Plotnick, Embedding homology 3-spheres in $S^{5} \ldots \ldots \ldots \ldots 147$

Norbert Riedel, Classification of the $C^{*}$-algebras associated with minimal rotations

Benedict Seifert, Combinatorial and geometric properties of weight systems of irreducible finite-dimensional representations of simple split Lie algebras over fields of 0 characteristic

James E. Simpson, Dilations on locally convex spaces

Paolo M. Soardi, Schauder bases and fixed points of nonexpansive mappings

Yoshio Tanaka, Point-countable $k$-systems and products of $k$-spaces

Fausto A. Toranzos, The points of local nonconvexity of starshaped sets . . . 209

Lorenzo Traldi, The determinantal ideals of link modules. I . . . . . . . . 215

P. C. Trombi, Invariant harmonic analysis on split rank one groups with applications

Shinji Yamashita, Nonnormal Blaschke quotients 